\title{
Resonance-enhanced multiple ionization of krypton at an x-ray free-electron laser
}

\author{
Benedikt Rudek, ${ }^{1,2,3, *}$ Daniel Rolles, $, 13,4, *$ Sang-Kil Son, ${ }^{5}$ Lutz Foucar, ${ }^{1,4}$ Benjamin Erk,,${ }^{1,2}$ Sascha Epp,,${ }^{1,2}$ Rebecca Boll, ${ }^{1,2}$ \\ Denis Anielski, ${ }^{1,2}$ Christoph Bostedt, ${ }^{6}$ Sebastian Schorb, ${ }^{6,7}$ Ryan Coffee, ${ }^{6}$ John Bozek, ${ }^{6}$ Sebastian Trippel, ${ }^{5}$ \\ Tatiana Marchenko, ${ }^{8}$ Marc Simon, ${ }^{8}$ Lauge Christensen, ${ }^{9}$ Sankar De,${ }^{10,11}$ Shin-ichi Wada, ${ }^{12}$ Kiyoshi Ueda, ${ }^{13}$ \\ Ilme Schlichting, ${ }^{1,4}$ Robin Santra, ${ }^{5,14}$ Joachim Ullrich, ${ }^{1,2,15}$ and Artem Rudenko ${ }^{1,2,16}$ \\ ${ }^{1}$ Max-Planck-Advanced Study Group at CFEL, DESY, Notkestraße 85, 22607 Hamburg, Germany \\ ${ }^{2}$ Max-Planck-Institut für Kernphysik, Saupfercheckweg 1, 69117 Heidelberg, Germany \\ ${ }^{3}$ DESY, Notkestraße 85, 22607 Hamburg, Germany \\ ${ }^{4}$ Max-Planck-Institut für medizinische Forschung, Jahnstraße 29, 69120 Heidelberg, Germany \\ ${ }^{5}$ Center for Free-Electron Laser Science CFEL, DESY, Notkestraße 85, 22607 Hamburg, Germany \\ ${ }^{6}$ SLAC National Accelerator Laboratory, 2575 Sand Hill Road, Menlo Park, California 94025, USA \\ ${ }^{7}$ Institut für Optik und Atomare Physik, Technische Universität Berlin, Hardenbergstraße 36, 10623 Berlin, Germany \\ ${ }^{8}$ Laboratoire de Chimie Physique-Matière et Rayonnement, UPMC and CNRS, 11 rue Pierre et Marie Curie, 75231 Paris, France \\ ${ }^{9}$ Department of Physics and Astronomy, Aarhus University, 8000 Aarhus C, Denmark \\ ${ }^{10}$ Department of Chemistry, Aarhus University, 8000 Aarhus C, Denmark \\ ${ }^{11}$ Saha Institute of Nuclear Physics, 700064 Kolkata, India \\ ${ }^{12}$ Department of Physical Science, Hiroshima University, 739-8236 Higashi-Hiroshima, Japan \\ ${ }^{13}$ IMRAM, Tohoku University, Katahira 2-1-1, Aoba-ku, 980-8577 Sendai, Japan \\ ${ }^{14}$ Department of Physics, Universität Hamburg, Jungiusstraße 9, 20355 Hamburg, Germany \\ ${ }^{15}$ Physikalisch-Technische Bundesanstalt, Bundesallee 100, 38116 Braunschweig, Germany \\ ${ }^{16}$ J. R. Macdonald Laboratory, Kansas State University, 116 Cardwell Hall, Manhattan, Kansas 66506, USA
}

(Received 18 January 2013; published 19 February 2013)

\begin{abstract}
The sequential inner-shell multiple ionization of krypton was investigated at the Linac Coherent Light Source using ion time-of-flight spectroscopy at photon energies above ( $2 \mathrm{keV})$ and below $(1.5 \mathrm{keV})$ the $L$ edge with two $\mathrm{x}$-ray pulse lengths ( 5 and $80 \mathrm{fs}$, nominally) and various pulse energies. At $2.5 \mathrm{~mJ}$ pulse energy, charge states up to $\mathrm{Kr}^{17+}$ were recorded for $M$-shell ionization and charge states up to $\mathrm{Kr}^{21+}$ for $L$-shell ionization. Comparing the experimental charge state distribution to Monte Carlo rate-equation calculations, we find a strong enhancement of higher charge states at $2 \mathrm{keV}$ photon energy as compared to the theoretical predictions. This enhancement is explained with a resonant ionization pathway where multiple excitations into outer valence and Rydberg orbitals are followed by autoionization. These resonant pathways play an important role for the photoionization of ions with charge higher than $\mathrm{Kr}^{12+}$, for which direct one-photon $L$-shell photoionization is energetically impossible at $2 \mathrm{keV}$ photon energy. Only a small pulse-length dependence of the charge state yield is observed at an $\mathrm{x}$-ray pulse energy of $0.4 \mathrm{~mJ}$.
\end{abstract}

DOI: 10.1103/PhysRevA.87.023413

PACS number(s): $32.80 . \mathrm{Rm}, 41.60 . \mathrm{Cr}, 32.80 . \mathrm{Zb}$

\section{INTRODUCTION}

The development of high-intensity short-pulsed x-ray sources promises revolutionary new techniques in diverse scientific fields, among others nurturing the vision of dynamic imaging of matter with angstrom spatial and femtosecond temporal resolution. Samples for these studies range from isolated atoms and small molecules to nanoparticles and solid targets [1,2]. As complex as the response of an extended system to the ultraintense femtosecond $\mathrm{x}$-ray pulses may be, the starting point is always a description on a microscopic, atomistic level, thus emphasizing the need for a clear picture of multiphoton ionization of individual atoms.

The first experiments at high $\mathrm{x}$-ray intensities and $\mathrm{keV}$ photon energies, which became feasible with the startup of the world's first $x$-ray free-electron laser (XFEL), the Linac Coherent Light Source (LCLS) [3], have shown that the dominant ionization mechanism under these conditions is

\footnotetext{
*Corresponding authors: benedikt.rudek@asg.mpg.de; daniel. rolles@asg.mpg.de
}

sequential multiphoton absorption [4,5]. It was demonstrated that all electrons from light elements like neon or nitrogen can be stripped in a single pulse provided the photon energy is higher than all ionization potentials of the intermediate ionic states. The rate of direct ("nonsequential") two-photon ionization has been shown to be small $[6,7]$ and its contribution to the total ionization yield can be neglected whenever sequential ionization is energetically allowed. Also, hidden resonant excitations were unveiled in high charge states of neon [8], but their role in the overall photoionization process is negligible for light atoms as they appear very localized. The creation of double-core-hole states was observed and has received considerable attention in the context of its application for chemical analysis [9-11]. In addition, experiments with different pulse durations revealed the phenomenon of frustrated absorption, or "intensity-induced x-ray transparency" reflecting efficient depletion of the $K$ shells of light elements in intense $\mathrm{x}$-ray pulses [4,5].

Our recent study on a much heavier atom, xenon, has shown that resonant excitations in intermediate ionic states can considerably enhance $x$-ray multiphoton ionization driving it well beyond the limit of direct single-photon transitions into 
the continuum [12]. These resonant pathways could nearly double the amount of energy absorbed by xenon atoms at $1.5 \mathrm{keV}$ photon energy, and, thus, have direct implications on the overall radiation damage of samples containing heavy atoms.

In this paper we report on the observation of resonanceenhanced X-ray multiple ionization (REXMI) of another high$Z$ element, krypton, by intense (up to $2.5 \mathrm{~mJ}$ ) LCLS pulses. We observe charge states up to $\mathrm{Kr}^{21+}$ at a photon energy of $2 \mathrm{keV}$, and up to $\mathrm{Kr}^{17+}$ at $1.5 \mathrm{keV}$, above and below the neutral krypton $L$ edges, respectively. Comparing the measured charge state distributions with the predictions of our theoretical model $[12,13]$, we find that the model, which does not take into account bound-bound (resonant) transitions, considerably underestimates the highest charge states produced at $2 \mathrm{keV}$. Since at $1.5 \mathrm{keV}$, where we do not expect any bound-bound transitions to play a role, the experimental data are in good agreement with theory, we interpret the discrepancy at $2 \mathrm{keV}$ as a signature of REXMI. From these data, as well as from our earlier results on xenon [12], we conclude that for such REXMI process to be efficient, the incident photon spectrum needs to cover resonant excitations in several subsequent charge states within the bandwidth of the XFEL pulse. Finally, comparing the measured and calculated charge state distributions for different pulse durations ( $\approx 5 \mathrm{fs}$ and $\approx 80 \mathrm{fs}$ ), we do not find any signatures of frustrated $\mathrm{x}$-ray absorption for krypton under our experimental conditions.

\section{EXPERIMENTAL SETUP}

The experiments were conducted in the CFEL-ASG multipurpose (CAMP) instrument [14] installed at the LCLS beamline for atomic, molecular and optical science (AMO) [15]. In this measurement campaign the CAMP setup was used as an ion time-of-flight and imaging spectrometer, and the pnCCD photon detectors were not installed [16]. After passing through a set of photoluminescence-based pulse-energy detectors [17] and an attenuation chamber used to adjust the beam energy for intensity-dependent measurement upstream of the AMO beamline, the LCLS beam $(120 \mathrm{~Hz}$ repetition rate, 1.5 and $2 \mathrm{keV}$ photon energies) was reflected on three steering mirrors, and focused inside the CAMP main chamber (background pressure $\approx 2 \times 10^{10}$ mbar) by a pair of two dynamically bent grazing-incidence mirrors in Kirkpatrick-Baez geometry [18]. The overall transmission of the beamline and the focal spot dimensions will be discussed below.

At the focal point the LCLS beam intercepted a thin supersonic gas jet of krypton atoms ( $4 \mathrm{~mm}$ FWHM). The produced ions were extracted by a homogeneous electric field created by evenly spaced, gold-plated aluminum electrodes. The extraction field was perpendicular to both the gas jet and LCLS beam propagation directions, whereas the LCLS beam polarization was parallel to the gas jet. The array of electrodes was divided into an acceleration region with constant voltage gradient and a drift region without voltage gradient. The two regions were physically separated by a copper grid to avoid penetrating fields. The extraction field in the 50-mm-long acceleration region was $10 \mathrm{~V} / \mathrm{cm}$ for measurements with "long" (nominally $80 \mathrm{fs}$ ) pulses and $400 \mathrm{~V} / \mathrm{cm}$ for runs with short $(\approx 5 \mathrm{fs})$ pulses. The 150 -mm-long drift zone was kept field-free. After the drift region the ions were postaccelerated to $2.45 \mathrm{keV}$ and projected onto a time-sensitive microchannel-plate (MCP) detector (in chevron configuration) with a delay-line anode mounted behind it for position detection. The spectrometer and detector geometry resulted in a $4 \pi$ acceptance for all atomic ions created within the overlap region between the LCLS beam and gas jet target.

The detector signals were recorded for each FEL shot with an Acqiris DC282 digitizer, and each ion hit was identified in postanalysis using a software constant-fraction discriminator [19] such that the effect of pulse-height variations due to varying MCP efficiency for different ionic charge states was negligible. Furthermore, the count rate was limited, on average, to ten $\mathrm{Kr}$ ions per pulse by adjusting the target density accordingly such that MCP pulses from individual ions could be detected in a counting mode and the number of overlapping MCP pulses was negligible. In addition to the time-of-flight (TOF) value obtained from the MCP trace, the hit position on the detector was reconstructed for each ion from the delay-line signals, which allowed us to completely eliminate the ions resulting from the residual gas or krypton diffused into the reaction chamber from those originating from the supersonic jet target and considered in the final TOF spectra.

In these experiments we used two different LCLS pulse durations, nominally 80 and $5 \mathrm{fs}$. Since these values are defined from the length of the electron bunch, they provide only a rough estimate of the actual temporal width of the X-ray pulse. For longer pulses, dedicated pulse-duration measurements [20] suggested that the actual $\mathrm{x}$-ray pulse might be considerably shorter than these nominal values, which appears plausible since the electron bunch length should give an upper limit for the $\mathrm{x}$-ray pulse duration. For the short (sub-10 fs) pulses, which are produced in a low bunch-charge mode, the simulations from the measured bunch parameters suggest that the x-ray pulse might be as short as a few femtoseconds [21]. With this in mind, we will, throughout the paper, nevertheless refer to the long and short pulses as $80 \mathrm{fs}$ and $5 \mathrm{fs}$, respectively.

\section{THEORETCAL APPROACH AND MODELING}

To simulate the ionization and relaxation dynamics induced by the LCLS X-ray pulse, we used the XATOM toolkit [22]. The version of XATOM used here is described in Refs. [12,13] and represents an extension of the model described in Refs. [4,23,24]. All X-ray-induced atomic processes were treated within a consistent theoretical framework. The orbital binding energies, photoionization cross sections, and Auger and Coster-Kronig rates for all possible $q$-hole electronic configurations of $\mathrm{Kr}^{q+}$ were calculated using the HartreeFock-Slater method. Shakeoff was included for neutral and singly charged krypton atoms, whereas double Auger decay and Auger satellites were not considered. Because the mean free path of electrons and ions in our very dilute gas target exceeded the gas jet size by several orders of magnitude, all collisional processes such as dielectric recombination, impact ionization, and charge exchange could be safely neglected. No resonant excitations to unoccupied, bound subshells and accompanying decay processes were considered here since this would enormously increase the total number of configurations. 
The calculated cross sections and rates are then used as input parameters for a set of rate equations for the time-dependent populations of the included configurations. Instead of solving the whole set of coupled rate equations directly, we used the Monte Carlo method [13], which follows probable pathways of ionization dynamics. Around 10 000-30 000 trajectories were calculated to converge the averaged populations. We assumed that, on average, the $\mathrm{x}$-ray temporal pulse shape was a Gaussian of $80 \mathrm{fs}$ (FWHM) or 5 fs duration. In the regime considered here, the results do not appear sensitive to the pulse shape or spikiness of individual FEL pulses and have only a very small dependence on the pulse duration such that actual x-ray pulses shorter than the nominal values do not significantly change the outcome of the calculations.

For each set of x-ray pulse parameters, the final charge state distributions were calculated. For a given pulse energy we determined the fluence on target by comparing benchmark calculations for neon atoms with experimentally determined neon charge state distributions measured at $2.0 \mathrm{keV}$ with the same pulse parameters as used for the krypton data. The results were integrated over the three-dimensional interaction volume defined by the intersection of two beams. This three-dimensional volume integration was not sensitive to the Rayleigh length $(\approx 10 \mathrm{~mm})$, unless the latter was set shorter than the gas jet diameter. We assumed a circular Gaussian x-ray beam profile and a circular Gaussian gas jet profile $(4 \mathrm{~mm}$ diameter, FWHM). Under this assumption, we determined a ratio between the beamline transmission and the focal spot size from the measured pulse energy and the fluence value calibrated with neon. Assuming a $3 \times 3 \mu \mathrm{m}^{2}$ focus (the best value ever measured in the CAMP chamber using the imprints technique [25]), we obtained a beamline transmission of $35 \%$ at $2.0 \mathrm{keV}$, which lies below the estimated reflectivity values for the transport mirrors as previously noted in Ref. [4].

\section{RESULTS AND DISCUSSION}

\section{A. Photon-energy dependence of the multiphoton ionization process}

The time-of-flight spectra for the unattenuated FEL beam at $80 \mathrm{fs}$ pulse duration, corresponding to a $2.4-2.6 \mathrm{~mJ}$ pulse energy interval at the gas detector, are depicted in Fig. 1(a) for photon energies of 1.5 [black (dark gray)] and 2 [red (light gray)] keV. Six krypton isotopes are distinguishable for low charge states with long flight times. For shorter flight times, the peaks from the isotopes of different charge states increasingly merge, but the most abundant isotope, ${ }^{84} \mathrm{Kr}$, can be separated to the highest charge state. The highest charge state observed at $1.5 \mathrm{keV}$ photon energy is $\mathrm{Kr}^{17+}$; at $2 \mathrm{keV}$ photon energy, it is $\mathrm{Kr}^{21+}$

To compare the ion spectra to calculations, the ${ }^{84} \mathrm{Kr}$ peak is integrated for every charge state. In Fig. 1(b), the calculated charge state distributions are shown as solid lines and the experimental data as bars. The ion yield for $1.5 \mathrm{keV}$ photon energy is drawn in black (dark gray) and for $2 \mathrm{keV}$ in red (light gray). Below the $L$ edge, at $1.5 \mathrm{keV}$, the highest yield is found for $\mathrm{Kr}^{3+}$ corresponding to single-photon $M$-shell ionization as observed at synchrotrons [26,27]. The predicted ion yield for $\mathrm{Kr}^{4+}$ is too low, probably due to the disregard of double
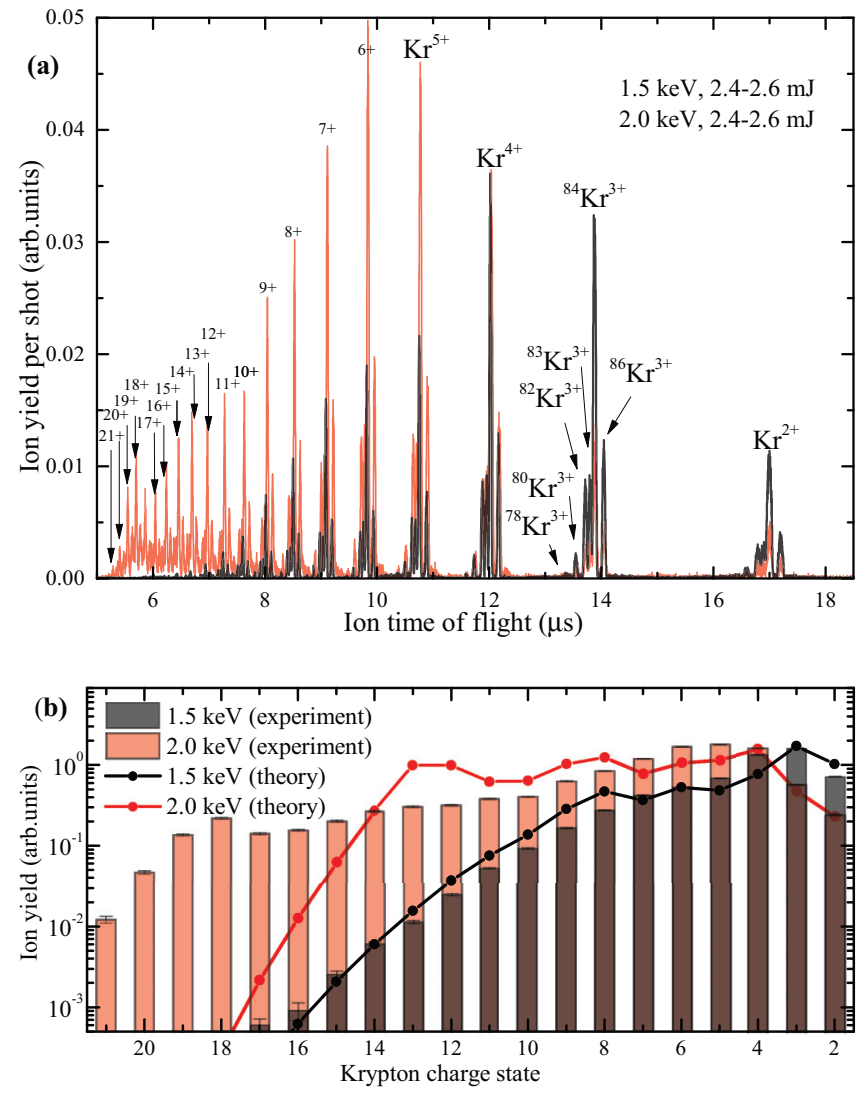

FIG. 1. (Color online) (a) Krypton time-of-flight spectra at photon energies above [2 keV, red (light gray)] and below [1.5 keV, black (dark gray)] the $L$ edge for nominally 80 fs pulses with $2.4-2.6 \mathrm{~mJ}$ pulse energy. (b) Experimental krypton charge state distribution (bars) and comparison to theory (circles with lines) calculated for an $80 \mathrm{fs}$ x-ray pulse with a pulse energy of $2.5 \mathrm{~mJ}$ and integrated over the interaction volume (see text). The theoretical charge state distributions are scaled such that the total ion yield integrated over all charge states agrees with the total ion yield in the experiment. Error bars for experimental data reflect the statistical uncertainties only.

Auger decay which occurs in $31 \%$ of the $3 d$ photoionization events [26]. For higher charge states, reached by absorption of additional photons, the theoretical and experimental data fit very well, indicating that the dominant processes are properly taken into account.

In the $2 \mathrm{keV}$ data, the highest ion yield is recorded for $\mathrm{Kr}^{5+}$ which, again, corresponds to single-photon absorption previously measured at synchrotrons [28,29]. Above $\mathrm{Kr}^{5+}$, the experimental ion yield continuously decreases (apart from a local peak at $\mathrm{Kr}^{18+}$ ), while the calculations show local maxima at $\mathrm{Kr}^{4+}, \mathrm{Kr}^{8+}$, and $\mathrm{Kr}^{12 / 13+}$. The peaks of $\mathrm{Kr}^{4+}$ and $\mathrm{Kr}^{8+}$ might be due to incompleteness of the model. The $\mathrm{Kr}^{12 / 13+}$ peak is due to the closing of direct $2 p$ photoionization at $\mathrm{Kr}^{12+}$. Apart from these differences at intermediate charge states, the most significant difference between experiment and calculations at $2 \mathrm{keV}$ is evident for the highest charge states produced, which are completely lacking in the calculations. For charge states higher than $\mathrm{Kr}^{12+}$, the only direct photoionization, which is energetically possible at $2 \mathrm{keV}$, is of the $M$ shell, so that the total photoionization cross section is significantly decreased. Additionally, at high intensities realized at free-electron lasers, 


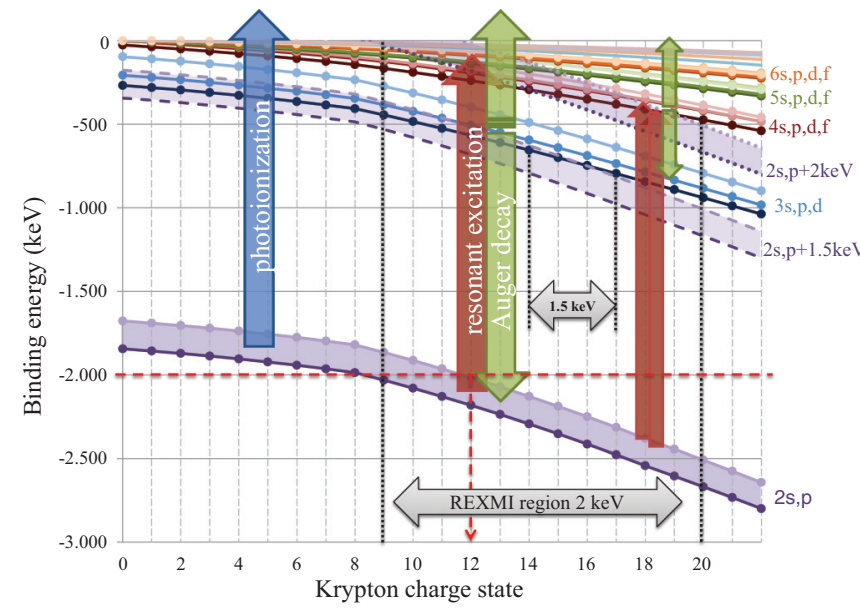

FIG. 2. (Color online) Ground-state binding energies for electrons in the $L, M$, and $N$ shells in krypton ions of a charge state up to $\mathrm{Kr}^{22+}$, together with schematic ionization pathways: direct photoionization is depicted as the blue (dark gray) arrow, resonant transitions as red (black) arrows, and Auger decay as green (light gray) arrows. To illustrate the occurrence of resonances at a photon energy of $2(1.5) \mathrm{keV}$, lines parallel to the $2 s$ and $2 p$ binding energies shifted by 2 (1.5) keV are drawn as purple dotted (dashed) lines. For charge states between $\mathrm{Kr}^{9+}$ and $\mathrm{Kr}^{20+}$, the dotted line intersects with the binding energies of various outer orbitals, hence demonstrating, from a purely energetic point of view, the possibility of resonant transitions from the $L$ shell to outer shells at $2 \mathrm{keV}$ photon energy. For $1.5 \mathrm{keV}$ photon energy, the region of energetically possible and electric-dipole-allowed resonances is limited to $\mathrm{Kr}^{14+}-\mathrm{Kr}^{17+}$.

double core holes can be created, when a second photon is absorbed before the initial inner-shell vacancy of the first photoionization is filled. Auger decay into such double (or multiple) $2 p$ holes and subsequent $M$-shell ionization are considered in the calculation, and charge states up to $\mathrm{Kr}^{17+}$ are predicted. The experimental distribution, however, shows significantly higher ion yields for those high charge states and a highest observed charge state of $\mathrm{Kr}^{21+}$. Apparently, an additional ionization pathway has to be considered beyond sequential multiphoton ionization.

The electronic ground-state configuration of neutral krypton is $1 s^{2} 2 s^{2} p^{6} 3 s^{2} p^{6} d^{10} 4 s^{2} p^{6}$. In Fig. 2, the orbital binding energies starting from the $2 s$ orbital up to the densely spaced Rydberg orbitals are plotted as functions of the ionic charge state. All binding energies increase with the higher charge states and they increase faster for inner shells. The wide blue arrow illustrates direct single-photon ionization of the $L$ shell, which dominates the ionization process for lower charge states. At $2 \mathrm{keV}$ photon energy, indicated by the horizontal dashed red line, direct photoionization of the $2 s$ orbital closes at $\mathrm{Kr}^{9+}$ and for the $2 p$ orbital at $\mathrm{Kr}^{12+}$, but resonant excitations [wide red (black) arrow] from the $L$ shell into Rydberg orbitals, which lie energetically very close together, open up instead. In order to illustrate where resonant transitions are energetically possible, purple dotted (dashed) lines parallel to the $2 s$ and $2 p$ binding energies but shifted by 2 (1.5) keV are drawn in Fig. 2. After resonant excitation, the resulting $L$-shell vacancy is filled via Auger decay, which increases the level of ionization by 1 . The region where any bound-bound transitions can occur is marked by two vertical dashed black lines in Fig. 2. For a photon energy of $1.5 \mathrm{keV}$, this region is small, and instead of excitation into densely spaced Rydberg and valence orbitals, the only energetically possible resonance is from $2 p$ to $3 s$ around $\mathrm{Kr}^{15+}$, where $3 s$, however, is occupied assuming the electronic ground-state configuration. Thus, resonant excitation plays a minor role at $1.5 \mathrm{keV}$ photon energy, but significantly enhances photoionization at $2 \mathrm{keV}$ photon energy, where resonant excitation into densely spaced outer shells can be found in a wide region from $\mathrm{Kr}^{9+}$ to $\mathrm{Kr}^{20+}$. It should be noted that in order to reach resonances in subsequent charge states within a single $x$-ray pulse, a relatively large bandwidth of the exciting $x$ rays is required. At LCLS, the unseeded, nonmonochromatized $\mathrm{x}$-ray beam has a bandwidth of $0.5 \%-1.0 \%$ of the photon energy, which is up to $20 \mathrm{eV}$ at $2 \mathrm{keV}$.

\section{B. Pulse-length dependence of the multiphoton ionization process}

In synchrotron experiments, where atomic ionization exclusively proceeds via single-photon absorption, the final charge state distributions are determined primarily by the photon energy. In contrast, at XFELs, where multiphoton processes become important, additional parameters such as pulse energy and pulse duration have to be considered. Here, besides the nonlinear dependence on the pulse energy, the absorption of multiple photons within one pulse can be influenced by the lifetime of inner-shell holes. In fact, first experiments at LCLS showed that the absorption probability is reduced when the X-ray pulse length is of the same order as the core-hole lifetime [4,5]. In the same way, resonance-driven multiple ionization might be suppressed, because resonant excitations can enhance or enable the photoionization processes only in conjunction with Auger decay.

To investigate the dependence of REXMI on the pulse duration, we measured ion charge state distributions at $2 \mathrm{keV}$ photon energy for 80 and 5 fs pulse durations (note that these are "nominal" values as discussed above). At the LCLS, pulse lengths below 10 fs require a low-charge operating mode (20 pC) [3], where the pulse energy is reduced to a maximum of $0.5 \mathrm{~mJ}$. To obtain the corresponding pulse energy with long pulses produced at full-charge mode for comparison, the $\mathrm{X}$-ray pulses were attenuated in the nitrogen-filled attenuation chamber upstream of the experimental setup.

Figure 3(a) displays the ion time-of-flight spectra of krypton irradiated with $\mathrm{x}$-ray pulses of $5 \mathrm{fs}$ [red (light gray)] and $80 \mathrm{fs}$ [black (dark gray)] pulse length, $2 \mathrm{keV}$ photon energy, and a pulse energy interval between 0.3 and $0.5 \mathrm{~mJ}$. Because the short-pulse data were recorded at higher spectrometer voltage $(2 \mathrm{kV}$ instead of $50 \mathrm{~V})$, the time of flight is shorter (as marked with the red axes on top and at right). In Fig. 3(b) the theoretical charge state distribution is depicted in circles along with the experimental distribution in bars derived from the TOF spectrum via integration of the ${ }^{84} \mathrm{Kr}$ peak.

Due to the reduced pulse energy, the maximum charge state with 5-fs pulses is $\mathrm{Kr}^{16+}$, while small yields up to $\mathrm{Kr}^{18+}$ can be observed in the 80 -fs data. As in Fig. 1, the most abundant species for both pulse durations is $\mathrm{Kr}^{5+}$ stemming from single-photon ionization. While theory agrees very well 

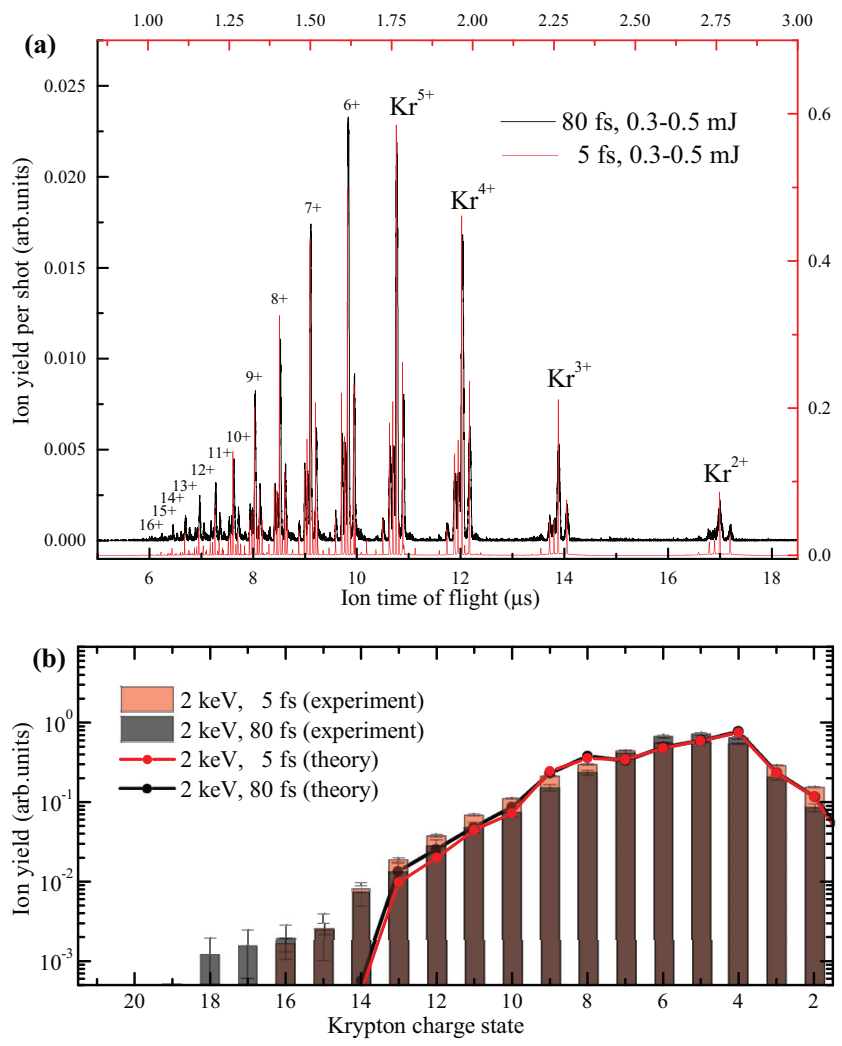

FIG. 3. (Color online) (a) Krypton TOF spectra at $2 \mathrm{keV}$ photon energy for nominally $80 \mathrm{fs}$ [black (dark gray)] and $5 \mathrm{fs}$ [red (light gray)] pulses with $0.3-0.5 \mathrm{~mJ}$ pulse energy. (b) Experimental krypton charge state distribution (bars) and comparison to theory (circles with lines) calculated for an 80 and a 5 fs x-ray pulse with a pulse energy of $0.4 \mathrm{~mJ}$ and integrated over the interaction volume. The theoretical charge state distributions are scaled such that the total ion yield integrated over all charge states agrees with the total ion yield in the experiment. Error bars for experimental data reflect the statistical uncertainties only.

with the experimental distribution for low charge states, it underestimates the yield of the highest charge states due to the disregard of the REXMI pathways, as was also found for the higher pulse energies shown in Fig. 1. Theoretically, no significant difference between the spectra for short and long pulses is predicted. Although this is seemingly at odds with the observations in other atoms and molecules [4,5], where suppressed absorption was observed for short x-ray pulses, it can be readily understood qualitatively considering the core-hole lifetimes in $\mathrm{Kr}$.

In Table I the Auger decay times in the Auger cascade following photoionization in the $2 s$ orbital of krypton are displayed. As can be seen from the table, $L$-shell vacancies are filled within less than $1 \mathrm{fs}$, much faster than both x-ray pulse durations. The subsequent $M N N$ Auger decay happens, on average, on the time scale of the short x-ray pulse, and only further decay steps involving the outer shell substantially exceed the short pulse length. In comparison, the Auger decay time of an $L$-shell vacancy in aluminum, where suppressed absorption was observed using 15-fs-long soft x-ray pulses [30], is $40 \mathrm{fs}$, while the $K$-shell lifetime in Ne, where similar effects where found in the $\mathrm{x}$-ray regime, is $2.4 \mathrm{fs}$ [31], and
TABLE I. Exemplary Auger cascade upon $2 s$ photoionization in krypton with average Auger decay times. For higher charge states, decay times increase, e.g., for the $2 s$ transition from 160 as in $\mathrm{Kr}^{0+}$ to 840.2 as in $\mathrm{Kr}^{9+}$ or to $2 \mathrm{fs}$ in $\mathrm{Kr}^{19+}$.

\begin{tabular}{ll}
\hline \hline Transition & Lifetime \\
\hline $\mathrm{Kr}^{+}\left(2 s^{-1}\right) \rightarrow \mathrm{Kr}^{2+}\left(2 p^{-1} 3 d^{-1}\right)$ & $\tau=160$ as \\
$\mathrm{Kr}^{2+}\left(2 p^{-1} 3 d^{-1}\right) \rightarrow \mathrm{Kr}^{3+}\left(3 d^{-3}\right)$ & $\tau=560$ as \\
$\mathrm{Kr}^{3+}\left(3 d^{-3}\right) \rightarrow \mathrm{Kr}^{4+}\left(3 d^{-2} 4 s^{-1} 4 p^{-1}\right)$ & $\tau=7 \mathrm{fs}$ \\
$\mathrm{Kr}^{4+}\left(3 d^{-2} 4 s^{-1} 4 p^{-1}\right) \rightarrow \mathrm{Kr}^{5+}\left(3 d^{-1} 4 s^{-2} 4 p^{-2}\right)$ & $\tau=18 \mathrm{fs}$ \\
$\mathrm{Kr}^{5+}\left(3 d^{-1} 4 s^{-2} 4 p^{-2}\right) \rightarrow \mathrm{Kr}^{6+}\left(4 s^{-2} 4 p^{-4}\right)$ & $\tau=155 \mathrm{fs}$ \\
\hline \hline
\end{tabular}

further rises with increasing charge state, e.g., to 23 fs at $\mathrm{Ne}$ $7+[4]$.

As the charge state increases, the core-hole lifetime in the $\mathrm{Kr} L$ shell increases as shown in Fig. 4, mainly because fewer valence electrons are available for the decay process. However, the core-hole lifetime is still shorter than the short $\mathrm{x}$-ray pulse duration for up to $\mathrm{Kr}^{23+}\left(2 s\right.$ single core hole) and $\mathrm{Kr}^{21+}$ ( $2 p$ single core hole). In order to produce $L$-shell multiplecore-hole states which accompany suppressed absorption, the $L$-shell photoabsorption process needs to occur earlier than the Auger decay does; in other words, the Auger lifetime needs to be comparable to or longer than the inverse of the $2 s$ or $2 p$ photoabsorption rate. The inverse of the $2 s$ photoabsorption rate is $\approx 40$ fs at the peak of the pulse with a pulse energy of $0.4 \mathrm{~mJ}$ and a pulse duration of $5 \mathrm{fs}$, and the inverse of the $2 p$ photoabsorption rate is $\approx 4 \mathrm{fs}$ in the same condition. The Auger lifetimes are much shorter than these time scales of photoabsorption (subfemtosecond from neutral krypton up to $\mathrm{Kr}^{10+}$ ). Thus, suppressed absorption does not play a role at the fluences used in this experiment. It should also be noted that because of the larger focal spot size in the CAMP instrument compared to the high-field physics end station used in [4,5], the peak fluence for a given pulse energy reached in this work is considerably lower than in [4,5], also reducing the frustrated $\mathrm{x}$-ray absorption effect.

Nevertheless, pulse-length-dependent effects may still occur since the REXMI process is sensitive also to the longer Auger decay times in the outer shells. However, this should be more significant at higher $\mathrm{x}$-ray fluences, where REXMI

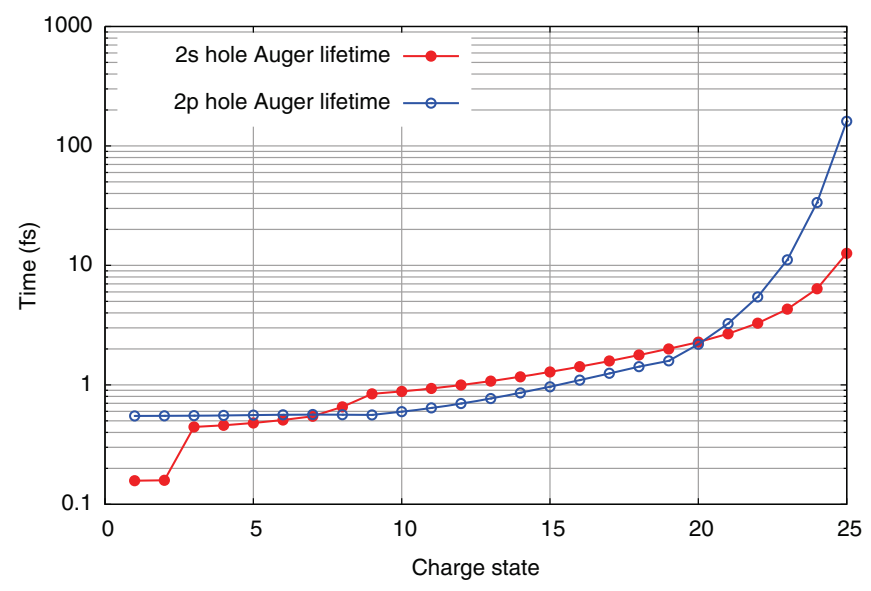

FIG. 4. (Color online) Auger lifetimes of $L$-shell single vacancy as a function of charge states. 
becomes more prominent (such as in the $2.5 \mathrm{~mJ}$ data shown in Fig. 1).

\section{Fluence dependence of the multiphoton ionization process}

Finally, the fluence dependence of the ionization processes was investigated for both pulse lengths. The fluence is calculated as the measured pulse energy multiplied by the transmission and divided by the focal area; due to an assumed Gaussian fluence distribution the result is multiplied by another factor of $4 \ln (2) / \pi$. Because intensity is defined as the fluence divided by the pulse length, the intensity is considerably higher for the shorter pulses. In Fig. 5 the ion yield per shot in logarithmic units is plotted against the peak fluence, likewise in logarithmic units. Because the unsaturated ion yield depends on the fluence to the power of $n$, with $n$ as the number of absorbed photons, the slope in a double-logarithmic graph gives the average number of photons that were absorbed in order to reach a certain charge state. Resonant transitions can influence the yield curves in two ways: first, they increase the cross section so that a given yield is already found at lower fluences than expected without resonances; second, they can saturate a transition so that it loses its fluence dependence. Then the slope decreases by the number of saturated transitions involved in the multiphoton ionization. For instance, this effect was observed at the soft-X-ray free-electron laser the SPring- 8 Compact SASE Source for resonances in helium [32].

In Fig. 5, the long-pulse data are illustrated by dots, the short-pulse data by stars, calculated yields are drawn as solid

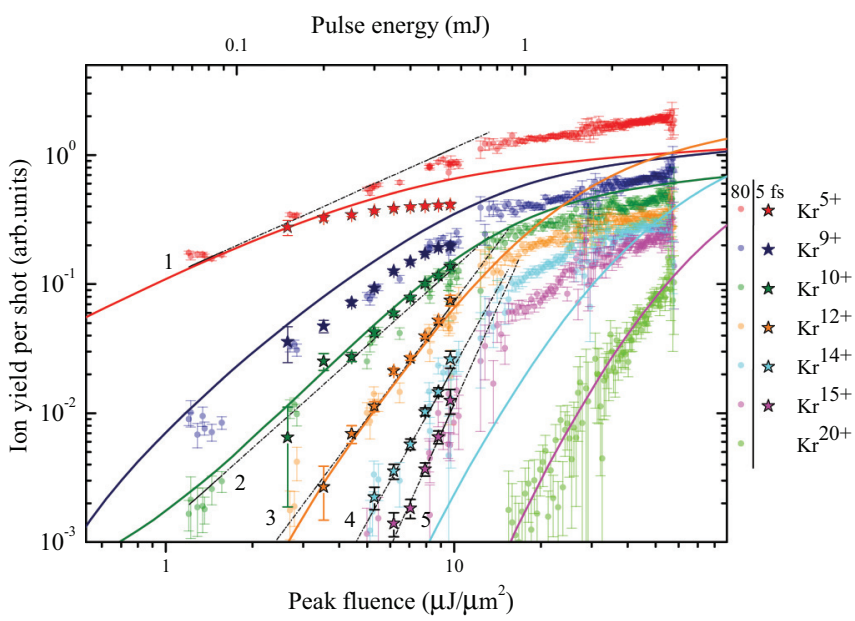

FIG. 5. (Color online) Krypton ion yield for selected charge states $\mathrm{Kr}^{q+}$ as a function of $\mathrm{x}$-ray fluence $\left(\mathrm{Kr}^{5+}\right.$ on top; higher charge states consecutively follow beneath). Measurements at a photon energy of $2.0 \mathrm{keV}$ are compared for nominal FEL pulse lengths of $80 \mathrm{fs}$ (dots) and $5 \mathrm{fs}$ (stars). The fluence at $2.0 \mathrm{keV}$ is calculated from the $\mathrm{x}$-ray pulse energy measured by the LCLS gas detectors (top axis) assuming a $3 \times 3 \mu \mathrm{m}^{2}$ focus and $35 \%$ beamline transmission. To correct for gas detector nonlinearities at FEL pulse energies below $0.8 \mathrm{~mJ}$, the gas detector readings were recalibrated using a linear ion signal (that is, $\mathrm{H}^{+}$ions created from residual gas). Calculated ion yields (without inclusion of REXMI) are shown as solid lines. To guide the eye, dashed lines with integer slopes are drawn through the experimental data points before they reach saturation. Error bars in the experimental data reflect statistical error only. lines, and dashed black lines with integer slopes are added to guide the eye. For short pulses, the LCLS pulse energy fluctuation is between 0.1 and $0.5 \mathrm{~mJ}$. Long pulses had a pulse energy between 2 and $3 \mathrm{~mJ}$ at full beam and were attenuated to distributions centered around 1.2, 0.5, 0.3, 0.15, and $0.07 \mathrm{~mJ}$. Because the LCLS pulse energy monitors seemed to be disturbed by attenuation pressures required to reach pulse energies below $0.8 \mathrm{~mJ}$, the pulse energy was recalibrated with the proton yield stemming from residual gas which is assumed to have a linear pulse energy dependence.

Seven exemplary krypton charge states are depicted in Fig. 5: the ion yield of $\mathrm{Kr}^{5+}$ is biggest so it is depicted as the topmost line, and higher charge states consecutively follow beneath. For $\mathrm{Kr}^{5+}$, the slope is 1 before the ion yield saturates, corresponding to single-photon ionization accompanied by a long Auger cascade. Other charge states up to $\mathrm{Kr}^{7+}$ (not depicted) also have a slope of 1 and saturate at similar fluence. Charge states such as $\mathrm{Kr}^{8+}, \mathrm{Kr}^{11+}$, or $\mathrm{Kr}^{13+}$ have a noninteger slope which lies between the slopes of the next lower and next higher charge states that are depicted here. The long-pulse data saturate at higher ion yield than the theory line, indicating that the experimental focus has more low-fluence areas than are simulated. The early saturation of the $\mathrm{Kr}^{5+}$ signal for short pulses is most likely an artifact of the MCP detector saturation appearing at high ion yield combined with high $(2 \mathrm{kV})$ extraction voltage used.

Two photons need to be absorbed to reach $\mathrm{Kr}^{9+}$ or $\mathrm{Kr}^{10+}$; $\mathrm{Kr}^{12+}$ requires the absorption of three photons according to the slopes in Fig. 5. The yield for $\mathrm{Kr}^{12+}$ at high fluence is lower than predicted, as the ionization to even higher charge states is enhanced by resonances. Also, the next higher charge states show signatures of resonant processes. Compared to the resonance-free calculations, the yield curve of $\mathrm{Kr}^{14+}$ is shifted by about $3 \mu \mathrm{J} / \mu \mathrm{m}^{2}$ to lower fluences due to the enhanced cross section, the curve of $\mathrm{Kr}^{15+}$ is shifted by about $20 \mu \mathrm{J} / \mu \mathrm{m}^{2}$. The slopes for those charge states lie between 4 and 5 . No significant differences in the slopes were found for short and long pulses.

\section{CONCLUSIONS}

In summary, we investigated the sequential inner-shell multiple ionization of krypton at photon energies above (2 $\mathrm{keV}$ ) and below (1.5 keV) the $L$ edge. We determined the number of sequentially absorbed photons to be one for $\mathrm{Kr}^{5+}$, two for $\mathrm{Kr}^{9+}$, three for $\mathrm{Kr}^{12+}$, and four for $\mathrm{Kr}^{14+}$. Below the $L$ edge, the experimental charge state distribution was adequately reproduced by theoretical calculations based on a rate-equation model. The same theoretical approach fails, however, at a photon energy above the $L$ edge, where the experimental spectrum shows higher charge states. We explain this enhancement by a resonance-enhanced $\mathrm{x}$-ray multiple ionization mechanism, i.e., resonant excitations followed by autoionization at charge states higher than $\mathrm{Kr}^{12+}$, where direct $L$-shell photoionization at $2 \mathrm{keV}$ is energetically closed. Interestingly, in xenon, where we recently studied multiphoton $\mathrm{x}$-ray ionization at the same two photon energies, we observed dramatic ionization enhancement due to REXMI at $1.5 \mathrm{keV}$, and achieved good agreement with theory at $2 \mathrm{keV}$. We readily understand this behavior since both photon energies are above 
the $M$ edge and below the $L$ edge in Xe, where we have shown that resonant excitations played a role only at $1.5 \mathrm{keV}$ under the given experimental conditions. The good agreement between theory and experiment in both cases, where we do not expect REXMI to play a role (Xe at $2 \mathrm{keV}, \mathrm{Kr}$ at $1.5 \mathrm{keV}$ ) demonstrates the reliability of the theoretical approach for these cases and also confirms our experimental parameters deduced from the comparison with the model simulations, in particular, the different beamline transmission and focus size at the two photon energies.

In contrast to earlier observations for lighter elements, a comparison of the 80 and 5 fs data showed no clear evidence of frustrated x-ray absorption or saturated resonances. The reasons for this are (i) subfemtosecond fast Auger decay of $L$-shell vacancies in $\mathrm{Kr}$; (ii) the higher number of electrons available at the $L$ shell compared to the $K$ shell; and (iii) the reduced intensity because of the larger focal spot size used in our experiment.

As discussed in [12], REXMI can significantly enhance the amount of energy absorbed per high- $Z$ atom. Since the data presented here confirm that REXMI is a rather general phenomenon for heavy atoms, this might have serious implications for local radiation damage in coherent diffractive imaging, in particular, in the vicinity of high- $Z$ impurities [16], if a photon energy is chosen where REXMI processes can occur. Because we expect REXMI-like processes to be efficient only for a rather broad bandwidth of the ionizing x-ray pulse, one important test would be a comparison between the ionization by a broadband and a narrowband pulse. Because of the recent success of the self-seeding scheme for hard $\mathrm{x}$ rays [33], this can be presently realized at the LCLS. A perfect candidate for such study would be the ionization of the xenon $L$ shell by the seeded and unseeded pulses in the microfocus of the LCLS coherent X-ray imaging (CXI) beamline.

\section{ACKNOWLEDGMENTS}

Portions of this research were carried out at the Linac Coherent Light Source (LCLS) at the SLAC National Accelerator Laboratory. The LCLS is an Office of Science User Facility operated for the US Department of Energy Office of Science by Stanford University. The authors acknowledge support from the Max Planck Society for funding the development and operation of the CAMP instrument within the Advanced Study Group at the CFEL. A.R. acknowledges support from Chemical Sciences, Geosciences, and Biosciences Division, Office of Basic Energy Sciences, Office of Science, US Department of Energy. K.U. acknowledges NEXT, the Japan Society for the Promotion of Science (JSPS), and the Institute of Multidisciplinary Research for Advanced Materials (IMRAM) and D.R. acknowledges support from the Helmholtz Gemeinschaft through the Young Investigator Program.
[1] J. Ullrich, A. Rudenko, and R. Moshammer, Annu. Rev. Phys. Chem. 63, 635 (2012).

[2] I. Schlichting and J. Miao, Curr. Opin. Struct. Biol. 22, 613 (2012).

[3] P. Emma et al., Nat. Photonics 4, 641 (2010).

[4] L. Young et al., Nature (London) 466, 56 (2010).

[5] M. Hoener et al., Phys. Rev. Lett. 104, 253002 (2010).

[6] G. Doumy et al., Phys. Rev. Lett. 106, 083002 (2011).

[7] A. Sytcheva, S. Pabst, S.-K. Son, and R. Santra, Phys. Rev. A 85, 023414 (2012).

[8] E. P. Kanter, B. Krässig, Y. Li, A. M. March, P. Ho, N. Rohringer, R. Santra, S. H. Southworth, L. F. DiMauro, G. Doumy, C. A. Roedig, N. Berrah, L. Fang, M. Hoener, P. H. Bucksbaum, S. Ghimire, D. A. Reis, J. D. Bozek, C. Bostedt, M. Messerschmidt, and L. Young, Phys. Rev. Lett. 107, 233001 (2011).

[9] L. Fang, M. Hoener, O. Gessner, F. Tarantelli, S. T. Pratt, O. Kornilov, C. Buth, M. Gühr, E. P. Kanter, C. Bostedt, J. D. Bozek, P. H. Bucksbaum, M. Chen, R. Coffee, J. Cryan, M. Glownia, E. Kukk, S. R. Leone, and N. Berrah, Phys. Rev. Lett. 105, 083005 (2010).

[10] N. Berrah, L. Fang, B. Murphy, T. Osipov, K. Ueda, E. Kukk, R. Feifel, P. van der Meulen, P. Salen, H. T. Schmidt, R. D. Thomas, M. Larsson, R. Richter, K. C. Prince, J. D. Bozek, C. Bostedt, S.-I. Wada, M. N. Piancastelli, M. Tashiro, and M. Ehara, Proc. Natl. Acad. Sci. USA 108, 16912 (2011).

[11] P. Salén, P. van der Meulen, H. T. Schmidt, R. D. Thomas, M. Larsson, R. Feifel, M. N. Piancastelli, L. Fang, B. Murphy, T. Osipov, N. Berrah, E. Kukk, K. Ueda, J. D. Bozek, C. Bostedt, S. Wada, R. Richter, V. Feyer, and K. C. Prince, Phys. Rev. Lett. 108, 153003 (2012).
[12] B. Rudek et al., Nat. Photonics 6, 858 (2012).

[13] S.-K. Son and R. Santra, Phys. Rev. A 85, 063415 (2012).

[14] L. Strüder, S. Epp, D. Rolles, R. Hartmann, P. Holl, G. Lutz, H. Soltau, R. Eckart, C. Reich, and K. Heinzinger, Nucl. Instrum. Methods Phys. Res., Sect. A 614, 483 (2010).

[15] J. D. Bozek, Eur. Phys. J.: Spec. Top. 169, 129 (2009).

[16] B. Erk, et al. Phys. Rev. Lett. 110, 053003 (2013).

[17] S. P. Hau Riege, R. M. Bionta, D. D. Ryutov, and J. Krzywinski, J. Appl. Phys. 103, 053306 (2008).

[18] A. Barty, R. Soufli, T. McCarville, S. L. Baker, M. J. Pivovaroff, P. Stefan, and R. Bionta, Opt. Express 17, 15508 (2009).

[19] L. Foucar, A. Barty, N. Coppola, R. Hartmann, P. Holl, U. Hoppe, S. Kassemeyer, N. Kimmel, J. Küpper, M. Scholz, S. Techert, T. A. White, L. Strüder, and J. Ullrich, Comput. Phys. Commun. 183, 2207 (2012).

[20] S. Düsterer et al., New J. Phys. 13, 093024 (2011).

[21] Y. Ding, A. Brachmann, F.-J. Decker, D. Dowell, P. Emma, J. Frisch, S. Gilevich, G. Hays, P. Hering, Z. Huang, R. Iverson, H. Loos, A. Miahnahri, H.-D. Nuhn, D. Ratner, J. Turner, J. Welch, W. White, and J. Wu, Phys. Rev. Lett. 102, 254801 (2009).

[22] S.-K. Son and R. Santra, XATOM-an integrated toolkit for X-ray and atomic physics, CFEL, DESY, Hamburg, Germany, 2011, Rev. 451.

[23] N. Rohringer and R. Santra, Phys. Rev. A 76, 033416 (2007).

[24] S.-K. Son, L. Young, and R. Santra, Phys. Rev. A 83, 033402 (2011).

[25] J. Chalupsky, P. Bohacek, V. Hajkova, S. Hau-Riege, P. Heimann, L. Juha, J. Krzywinski, M. Messerschmidt, S. Moeller, 
B. Nagler, M. Rowen, W. Schlotter, M. Swiggers, and J. Turner, Nucl. Instrum. Methods Phys. Res., Sect. A 631, 130 (2011).

[26] M. O. Krause and T. A. Carlson, Phys. Rev. 149, 52 (1966).

[27] G. S. Lightner, R. J. Van Brunt, and W. D. Whitehead, Phys. Rev. A 4, 602 (1971).

[28] Y. Morishita, Y. Tamenori, K. Okada, T. Oyama, K. Yamamoto, K. Tabayashi, T. Ibuki, K. Moribayashi, and I. H. Suzuki, J. Phys. B 39, 1323 (2006).
[29] M. O. Krause and T. A. Carlson, Phys. Rev. 158, 18 (1967). [30] B. Nagler et al. Nat. Phys. 5, 693 (2009).

[31] M. O. Krause, J. Phys. Chem. Ref. Data 8, 307 (1979).

[32] T. Sato, A. Iwasaki, K. Ishibashi, T. Okino, K. Yamanouchi, J. Adachi, A. Yagishita, H. Yazawa, F. Kannari, M. Aoyma, K. Yamakawa, K. Midorikawa, H. Nakano, M. Yabashi, M. Nagasono, A. Higashiya, and T. Ishikawa, J. Phys. B 44, 161001 (2011).

[33] J. Amann et al., Nat. Photonics 6, 693 (2012). 